\title{
Assessment of Inflammatory Status Based on Total Blood Count and Its Relationship to Cardiovascular Diseases
}

\author{
Anna-Boróka Tusa1, Cristian Podoleanu1,2, Zsuzsanna Szász²,3, Annamária Magdás1,2 \\ 1 Department of Internal Medicine IV, County Clinical Hospital Mureș, Târgu Mureș, Romania \\ 2 University of Medicine, Pharmacy, Science and Technology, Târgu Mureș, Romania \\ ${ }^{3}$ Department of Internal Medicine I, County Emergency Hospital Mureș, Târgu Mureș, Romania
}

\section{CORRESPONDENCE}

\section{Cristian Podoleanu}

Str. Gheorghe Marinescu nr. 38

540139 Târgu Mureș, Romania

Tel: +40 265215551

E-mail: podoleanu@me.com

\section{ARTICLE HISTORY}

Received: November 17, 2019

Accepted: December 14, 2019
Anna-Boróka Tusa • Str. Gheorghe Marinescu nr. 1 , 540103 Târgu Mures, Romania. Tel: +40 265 212111, E-mail: tusa.annaa@gmail.com

Zsuzsanna Szász • Str. Gheorghe Marinescu nr. 38, 540139 Târgu Mureș, Romania. Tel: +40 265 215551, E-mail: zsuzsannaagnes@yahoo.com

Annamária Magdás • Str. Gheorghe Marinescu nr. 38 540139 Târgu Mureș, Romania. Tel: +40 265215133 (212),E-mail: annamaria.magdas@umftgm.ro

\begin{abstract}
Background: Inflammation seems to be the most important trigger for atherosclerosis. Lower extremity artery disease (LEAD) and hypertension (HTN) are the most important atherosclerotic diseases. Total blood count-derived neutrophil-lymphocyte ratio (NLR) and red cell distribution width (RDW) have been proposed as inflammatory biomarkers in cardiovascular diseases. The aim of this study was to assess the correlation with LEAD and HTN. Material and methods: In total, 134 subjects were included in the study, divided into 2 groups as follows: group 1 - patients with LEAD and HTN; group 2 - subjects with HTN, without LEAD. Complete blood count (CBC) and lipid profile were determined. To assess peripheral artery disease, the ankle-brachial index (ABI) was measured with a handheld Doppler device (BiDop ES100V3 Hadeco'). We compared the inflammatory status between the groups with the use of white blood cell count (WBC), red cell distribution (RDW), and neutrophil-lymphocyte ratio (NLR). Results: In group 1, there was a significant negative correlation between the ABI and RDW ( $p=0.04, r^{2}=-0.24$, 95\% Cl: $0.4647-$ $0.0013)$, as well as between the ABI and NLR ( $\left.p=0.001, r^{2}=-0.38,95 \% \mathrm{Cl}: 0.5801-0.1592\right)$. In group 2, there was a positive correlation between NLR and total serum cholesterol levels $\left(p=0.003,95 \% \mathrm{Cl}: 0.1274-0.5472, r^{2}=0.12\right)$, as well as between NLR and triglyceride levels ( $p$ $\left.=0.002,95 \% \mathrm{Cl}: 0.1387-0.5552, r^{2}=0.13\right)$. Conclusion: NLR and RDW could represent first-line investigations in patients with cardiovascular disease due their cost efficiency. They can also play a role in triaging patients with atherosclerotic disease, monitoring treatment response and prognosis of the disease.
\end{abstract}

Keywords: inflammatory biomarkers, neutrophil-lymphocyte ratio, red cell distribution width, cardiovascular diseases

\section{INTRODUCTION}

The relation between inflammation and atherosclerotic disease is still a hot topic in the medical field. ${ }^{1}$ Peripheral artery disease (PAD) and hypertension (HTN) are the most frequent manifestations of atherosclerotic disease. ${ }^{2}$ Given the high cost associated with the assessment of inflammatory markers, some simple labo- 
ratory tests have been suggested to be performed as part of the routine clinical investigation. Neutrophil-lymphocyte ratio (NLR) and red cell distribution width (RDW) were related to risk prediction in PAD as well as in the development of HTN. ${ }^{1}$ RDW is a measure of the range of variation of red blood cell volume. The release of inflammatory cytokines may affect bone marrow function and consequently erythrocyte maturation, resulting in elevated RDW levels. ${ }^{3}$ The inflammatory mediators produced by neutrophils are associated with vascular stiffening, while the inflammatory response mechanisms are regulated by lymphocytes, showing an anti-atherosclerotic effect. Therefore, NLR has been proposed as a low-cost inflammatory biomarker. ${ }^{2}$ Studies reveal that higher NLR values are linked to higher amputation risk in lower extremity artery disease (LEAD), ${ }^{4}$ while $\mathrm{RDW}$ is associated with poor clinical outcomes in patients with cardiovascular diseases. ${ }^{3}$

Therefore, the aim of this study was to compare the inflammatory status of patients with lower extremity artery disease (LEAD) and HTN versus a control group consisting of hypertensive patients without LEAD.

\section{MATERIAL AND METHOD}

This retrospective study was performed at the Department of Internal Medicine IV of the Clinical County Hospital of Târgu Mureș between January 2018 and September 2018. A total of 134 subjects were included in the study, using the following inclusion criteria: history of HTN or use of hypertension medication, or office blood pressure (BP) values $>140 / 90 \mathrm{mmHg}$; LEAD or ankle-brachial index (ABI) $<0.9$, age $>18$ years. ${ }^{5}$ Patients with diabetes mellitus, kidney and liver disease, age $<18$ years, thrombosis, hematological disorders, malignancies, inflammatory diseases that could alter white blood cells (WBC), as well as pregnant women were excluded from the study. The patients were divided into two groups: group 1 included patients with LEAD and HTN, while group 2 included subjects without LEAD and with HTN. Patient characteristics were recorded in a standardized data collection form. As part of routine investigations, blood samples were collected in the morning after at least 8 hours of fasting including complete blood count (CBC), determined by a CELL-DYN Ruby Hematology Analyzer (Abbott Diagnostics ${ }^{\circ}$ ) using the flow cytometric technique, and lipid profile, assessed by an ARCHITECT c4000 Clinical Chemistry Analyzer (Abbott Diagnostics ${ }^{\circ}$ ). We compared the inflammatory status of the groups by analyzing WBC, RDW, and NLR (obtained by dividing the percentage of neutrophils by those of lymphocytes).

Statistical analysis was performed using the statistical program GraphPad Prism version 6. The KolmogorovSmirnov test was applied to test the normality of the study variables. Quantitative variables were compared using unpaired t-tests. Categorical variables are represented as mean \pm standard deviation (SD). To assess correlations between walking distance and RDW, Pearson's test was performed. The significance level was set at $95 \%$, and an a value $\leq 0.05$ was considered statistically significant.

A written informed consent was obtained from all patients included in the study, and all procedures were performed according to the ethical principles stated in the Declaration of Helsinki. The study was also approved by the ethics committee of the hospital.

\section{RESULTS}

Characteristics of the subjects are listed in Table 1.

In group 1, there was a significant negative correlation between the ABI and RDW (95\% CI: 0.4647-0.0013), shown in Figure 1, as well as between the ABI and NLR (95\% CI: 0.5801-0.1592), shown in Figure 2. A statistically significant positive correlation was recorded in this

TABLE 1. Demographic and laboratory data of the study population

\begin{tabular}{lccc}
\hline Variables & $\begin{array}{c}\text { Group 1 } \\
\text { (HTN +, LEAD +) }\end{array}$ & $\begin{array}{c}\text { Group 2 } \\
\text { (HTN +, LEAD -) }\end{array}$ & p value \\
\hline Age, years & $66.97 \pm 8.38$ & $66.12 \pm 11.31$ & 0.73 \\
Gender, M/F, n & $48 / 16$ & $30 / 40$ & 0.0002 \\
Total cholesterol, mg/dL & $185.2 \pm 6.61$ & $194.6 \pm 5.41$ & 0.26 \\
Triglycerides, mg/dL & $162.1 \pm 11.29$ & $155.5 \pm 11.67$ & 0.68 \\
Serum creatinine, mg/dL & $0.94 \pm 0.23$ & $0.93 \pm 0.23$ & 0.84 \\
WBC & $8.31 \pm 0.24$ & $6.76 \pm 0.19$ & $<0.001$ \\
Hemoglobin, g/dL & $13.88 \pm 0.27$ & $13.54 \pm 0.20$ & 0.32 \\
RDW, \% & $13.4 \pm 0.23$ & $12.78 \pm 0.15$ & 0.02 \\
NLR & $2.39 \pm 0.14$ & $2.03 \pm 0.09$ & 0.04 \\
\hline
\end{tabular}




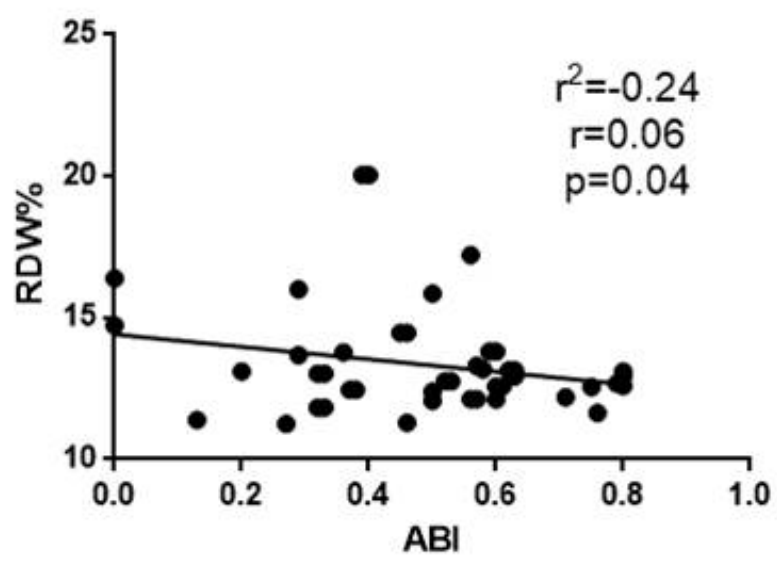

FIGURE 1. Correlation between RDW and the $A B I$ in group 1

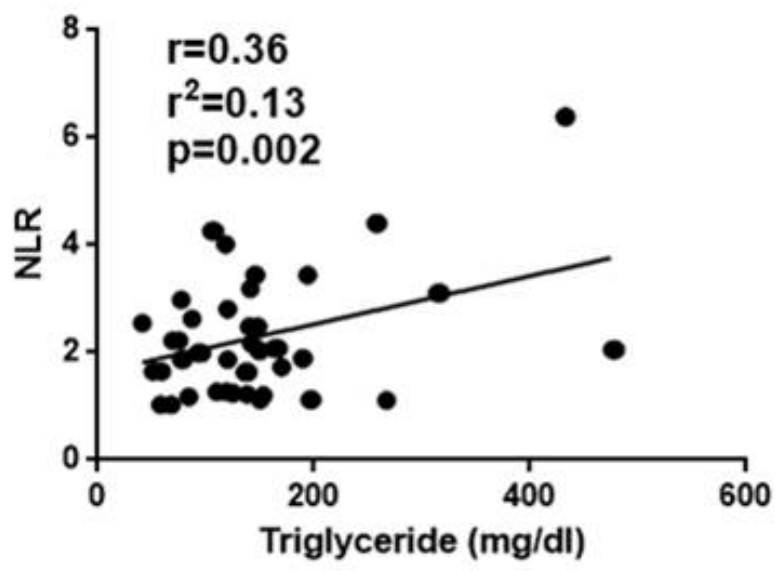

FIGURE 3. Correlation between NLR and triglyceride levels in group 2

group between NLR and total cholesterol $\left(\mathrm{p}=0.03, \mathrm{r}^{2}=\right.$ 0.04, 95\% CI: 0.0195-0.4789), as well as between NLR and triglyceride levels ( $\mathrm{p}=0.01, \mathrm{r}^{2}=0.08,95 \%$ CI: $0.05078-$ 0.5026). RDW was positively correlated with the level of trigly-cerides ( $\mathrm{p}=0.02, \mathrm{r}^{2}=0.06,95 \% \mathrm{CI}: 0.009-0.4710$ ) and total cholesterol (95\% CI: $0.5429-0.1059, \mathrm{p}=0.005$, $\left.\mathrm{r}^{2}=-0.34, \mathrm{r}=0.11\right)$.

In group 2, a positive correlation was found between NLR and total serum cholesterol levels $(p=0.003$, 95\% CI: $0.1274-0.5472, \mathrm{r}^{2}=0.12$ ), as well as between NLR and triglyceride levels ( $\mathrm{p}=0.002$; 95\% CI: 0.1387-0.5552, $\mathrm{r}^{2}=0.13$ ) (Figure 3 ). There was no significant correlation between RDW and serum triglyceride levels ( $p>0.05$ ). Also, we found no significant relationship between total cholesterol levels and RDW in this group, as shown in Figure 4 (95\% CI: $-0.1202-0.3501$ ).

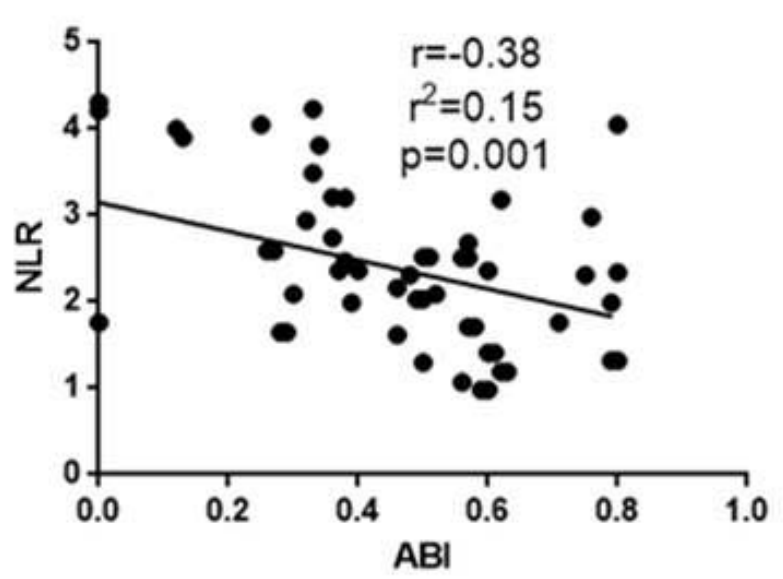

FIGURE 2. Pearson's correlation between NLR and the ABI in group 1

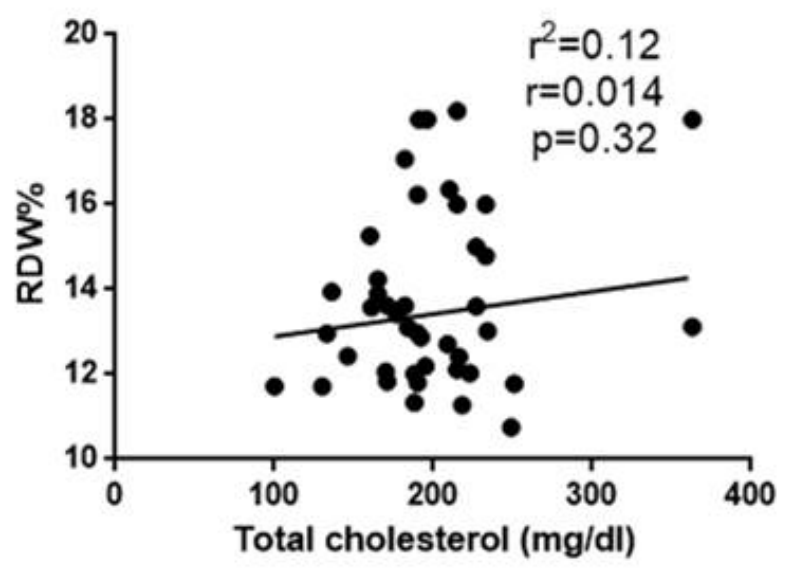

FIGURE 2. Correlation between RDW and total cholesterol levels in group 2

\section{DISCUSSIONS}

In this study, we aimed to investigate the relationship between the proposed inflammation markers, derived from total blood count, and cardiovascular risk factors. The prevalence of cardiovascular diseases is increasing. Therefore, researchers are targeting new biomarkers, which may have limited use due to their high cost of implementation. Recent data reveal some low-cost inflammation markers that could contribute to the progression of atherosclerosis. Their assessment could represent a first step in the triage of patients for further costly investigations. We found that patients with LEAD and HTN had greater levels of white blood cells. In this group, the levels of inflammatory markers, such as NLR and RDW, were higher. In retrospective studies, it was shown that greater levels of NLR were associated with a higher risk of developing critical limb ischemia and a greater risk of 
limb amputation in peripheral artery disease. ${ }^{6}$ In these studies, the cut-off value was defined at an NLR greater than 3.2.7 Although in our study groups this level was lower, 2.39 versus 2.03 , due to the fact that patients with critical ischemia or amputation were excluded, the difference was statistically significant toward the group associating two atherosclerotic diseases. In group $1(\mathrm{LEAD}+\mathrm{HTN})$, there was a negative but statistically significant correlation between NLR and the ABI, as well as between RDW and the ABI. This could be explained by the progression of atherosclerosis, produced by inflammation. In order to confirm these data, further inflammation-specific serological investigations are required. A study revealed that patients with LEAD with an RDW value above $14.5 \%$ were at higher risk of mortality compared to those with a RDW $<12.8 \% .{ }^{8}$ In group 2 (HTN without LEAD), only NLR was correlated with serum lipid levels, while RDW values were not; these findings are in accordance with the results of other researchers, who have found that higher NLR levels are observed even before the occurrence of target organ damage. Elevated NLR has been observed also in subjects susceptible for developing HTN, as well as in HTN patients with non-dipper profile. ${ }^{9}$ The increased proportion of neutrophils has been linked to an alteration of the immunologic response, which in turn is associated with endothelial dysfunction and oxidative stress. ${ }^{10}$ Nonetheless, NLR has been found to correlate with validated inflammatory markers such as C-reactive protein. ${ }^{11}$

This study has a couple of limitations. On the one hand, coronary artery disease was excluded based only on medical history and ECG records. On the other hand, we evaluated only total blood count-derived hematological markers in relation with cardiovascular diseases, and we did not include specific markers such as high-sensitivity C-reactive protein.

\section{CONCLUSION}

In conclusion, the widely used total blood count-derived parameters NLR and RDW seem to reflect the severity of atherosclerosis. They are cost-efficient, simple to use, and thus could represent the first investigations in patients with suspected or manifest cardiovascular disease. These biomarkers are also suitable for the triage of patients with atherosclerotic disease, to monitor treatment response and prognosis of the disease. Further studies are needed to correlate these data with more specific inflammatory markers, and cut-off values should be defined for specific population groups.

\section{CONFLICT OF INTEREST}

Nothing to declare.

\section{REFERENCES}

1. Spanos K, Matsagkas M, Giannoukas A. Full Blood Count and Peripheral Arterial Disease Severity and Prognosis. Curr Vasc Pharmacol. 2017;15:9495.

2. Paquissi FC. The role of inflammation in cardiovascular diseases: the predictive value of neutrophil-lymphocyte ratio as a marker in peripheral arterial disease. Ther Clin Risk Manag. 2016;12:851-860.

3. Duman E, Kulaksızoglu S, Çifçi E, Ozulku M. Is there a Real Correlation between Red Cell Distribution Width and Peripheral Arterial Disease? J Med Biochem. 2017;36:309-313.

4. Shere A, Eletta O, Goyal H. Circulating blood biomarkers in essential hypertension: a literature review. J Lab Precis Med. 2017;2:99.

5. Mancia G, Fagard R, Narkiewicz K, et al. 2013 ESH/ESC Guidelines for the management of arterial hypertension: The Task Force for the management of arterial hypertension of the European Society of Hypertension (ESH) and of the European Society of Cardiology (ESC). Eur Heart J. 2013;34:21592219

6. Belaj K, Pichler M, Hackl G, et al. Association of the Derived NeutrophilLymphocyte Ratio With Critical Limb Ischemia. Angiology. 2015;67:350354.

7. Taşoğlu I, Çiçek OF, Lafci G, et al. Usefulness of neutrophil/lymphocyte ratio as a predictor of amputation after embolectomy for acute limb ischemia. Ann Vasc Surg. 2014;28:606-613.

8. Ye Z, Smith C, Kullo IJ. Usefulness of red cell distribution width to predict mortality in patients with peripheral artery disease. Am J Cardiol. 2011;107:1241-1245.

9. Liu X, Zhang Q, Wu H, et al. Blood Neutrophil to Lymphocyte Ratio as a Predictor of Hypertension. Am J Hypertens. 2015;28:1339-1346.

10. Kotani K. Neutrophil/lymphocyte ratio and the oxidative stress burden. Can J Cardiol. 2015;31:365.e9.

11. Oh BS, Jang JW, Kwon JH, et al. Prognostic value of C-reactive protein and neutrophil-to-lymphocyte ratio in patients with hepatocellular carcinoma. BMC Cancer. 2013;13:78. 